This is a post-peer-review, pre-copyedit version of:

Cañas J.J., Ferreira P., López de Frutos P., Puntero E., López E., Gómez-Comendador F., de Crescenzio F., Lucchi F., Netjasov F., Mirkovic B. (2019) Mental Workload in the Explanation of Automation Effects on ATC Performance. In: Longo L., Leva M. (eds) Human Mental Workload: Models and Applications. H-WORKLOAD 2018. Communications in Computer and Information Science, vol 1012. Springer, Cham.

The final authenticated version is available online at: https://doi.org/10.1007/978-3$\underline{030-14273-5 \quad 12}$

This version is subjected to Springer Nature terms for reuse that can be found at: https://www.springer.com/gb/openaccess/authors-rights/aam-terms-v1 


\title{
Mental Workload in the Explanation of Automation Effects on ATC Performance
}

\author{
José Juan Cañas ${ }^{1(\bowtie)}$, Pedro Ferreira ${ }^{1}$, Patricia López de Frutos ${ }^{2}$, \\ Eva Puntero ${ }^{2}$, Elena López ${ }^{2}$, Fernando Gómez-Comendador ${ }^{3}$, \\ Francesca de Crescenzio ${ }^{4}$, Francesca Lucchi $^{4}$, Fedja Netjasov ${ }^{5}$, \\ and Bojana Mirkovic ${ }^{5}$ \\ ${ }^{1}$ Mind, Brain, and Behaviour Research Centre, University of Granada, \\ Granada, Spain \\ \{delagado, pedroferreira\}@ugr.es \\ 2 CRIDA A.I.E ATM R\&D + Innovation Reference Centre, Madrid, Spain \\ \{pmldefrutos, epuntero, elopezb\}@e-crida.enaire.es \\ 3 Aerospace Systems, Air Transport and Airports Department, \\ Polytechnic University of Madrid, Madrid, Spain \\ fernando.gcomendador@upm.es \\ 4 Industrial Engineering Department, University of Bologna, Forlì, Italy \\ \{francesca.decrescenzio, f. lucchi\}@unibo.it \\ ${ }^{5}$ Faculty of Transport and Traffic Engineering, University of Belgrade, \\ Belgrade, Serbia \\ \{f.netjasov,b.mirkovic\}@sf.bg.ac.rs
}

\begin{abstract}
Automation has been introduced more and more into the role of air traffic control (ATC). As with many other areas of human activity, automation has the objective of reducing the complexity of the task so that performance is optimised and safer. However, automation can also have negative effects on cognitive processing and the performance of the controllers. In this paper, we present the progress made at AUTOPACE, a European project in which research is carried out to discover what these negative effects are and to propose measures to mitigate them. The fundamental proposal of the project is to analyse, predict, and mitigate these negative effects by assessing the complexity of ATC in relation to the mental workload experienced by the controller. Hence, a highly complex situation will be one with a high mental workload and a low complex situation will be one in which the mental workload is low.
\end{abstract}

Keywords: Automation $\cdot$ Air-traffic controllers $\cdot$ Mental workload

\section{Introduction}

The control of complex and dynamic environments is a risky and uncertain task [1]. A complex and dynamic environment is one in which a person has to find a solution by performing a series of operations that can be characterised as follows: the number of elements relevant to the solution process is large and these are highly interconnected; the system changes dynamically over time; part of the structure and dynamics of the system are opaque (not transparent) for the operator; and the objectives can be multiple 
and sometimes conflicting [2-4]. Complexity is one of the most important causes of mental workload in many areas of human performance [5-10].

Air traffic control (ATC) has all the characteristics of a complex and dynamic task: there are many elements (aircrafts and obstacles) that are interconnected; all these elements are changing constantly due to both the actions of the air traffic controller (ATCo) and their own dynamics; some of the variables are not transparent to the ATCo; and the objective is to direct all aircraft traffic safely and orderly at the same time. For this reason, it has been indicated that the mental workload of the ATCo is related to the complexity of the ATC system [11,12]. A great deal of theoretical and applied research has been directed over the years to reducing the complexity of the ATCo task and, thus, to make it safer and less uncertain. In this research study, the focus has been mainly on the design of automation tools to which part of the control task can be allocated. The results of introducing new automatic tools in the control task have been mainly successful, but - similar to other areas of human work - automation also has some negative effects on system performance and safety [13, 14]. Those negative effects have motivated further research, with the aim of better understanding the impact of automation on the ATCo. The goal of this research is to improve the benefits and to reduce the negative effects of automation. With this goal, project AUTOPACE (Grant 699238), funded by the SESAR joint undertaking as part of SESAR 2020 Exploratory Research Programme within the framework of the EU's Horizon 2020, was intended to carry out scientific research to address the effects of high automation on ATCos' performances. At AUTOPACE, we assume that high automation will have effects on the level of mental workload experienced by the ATCo. Those effects could have positive as well as negative consequences on ATCo performance. To determine those consequences, in the project we propose research, using a psychological model based on established theories of attentional resources, to predict the effects of automation on ATCo mental workload. This model, which is designed to reduce complexity, could be used as the basis of investigation into the required new competences and training strategies which ensure that the ATCo's mental workload levels are compatible with the requirements of safe operation. A safe operation implies that the controller, in their new role which requires supervision and monitoring, remains 'in-the-loop' to initiate an efficient decision-making process, especially when dealing with possible unforeseen operational conditions and malfunctions of automation. A safe operation would also imply that the controller performance would not be affected by stressful situations when the system fails. Unforeseen operational conditions and malfunction of automation could lead to disorientation and panicked behaviour.

In the following sections, we will discuss the approach taken in the AUTOPACE project to the problems associated to reducing the mental workload by introducing automation in the ATCo task. The main assumption of the AUTOPACE project is that workload is related to the complexity of the system in which the ATCo performs their work. Therefore, we start by explaining in Sect. 2 our vision of what complexity means within the field of ATC and ATM in general. Next, in Sect. 3, we will expose the fundamental problem that the AUTOPACE project wants to address: the possible negative effects of automation on the performance of the ATCo. It is true that automation reduces the mental workload by reducing the demand for mental resources. 
However, automation can also cause phenomena such as out-of-the-loop or erratic behaviour when the automatic system fails. In consequence, in the AUTOPACE project there is a proposal to reduce complexity and mental workload by considering the negative effects of automation. This proposal is explained in Sect. 4. First, in Sect. 4 we expose the psychological model that we are developing based on the concept of mental workload. Then, in Sect. 5 we address the negative effects that we hypothesize that will be found in the future scenarios in ATC in which more automation will be introduced. In Sect. 6 we describe the methodology we are using to test the hypotheses contemplated in AUTOPACE. This methodology is based on the development of a computational model in which the psychological model of ATCo is implemented. In Sect. 6 some results obtained in the execution of this computational model are also presented. Finally, in the conclusions of the paper we indicate some consequences that the results of this project will have on the training of the ATCo designed to face the new automation scenarios.

\section{Approaches to Defining and Measuring Complexity in ATC}

Complexity features as a topic of scientific research and theory in many academic and applied fields [15]. However, a review of this research shows that all approaches to the complexity issue start from an explicit or implicit definition of what is meant by complexity. In particular, in the field of ATM (air traffic management) we can find three approaches in which complexity is defined and measured differently.

\subsection{The Algorithmic Approach}

In the algorithmic approach (see Fig. 1a), it is assumed that the complexity of the task can be calculated directly from the parameters of the environment (i.e., [16]). Thus, over the years, some formulas have been proposed in which complexity is calculated from parameters such as occupancy (number of aircraft in the sector) or the meteorological conditions. In this approach, the controller is not taken into account in the definition of complexity. Complexity is defined only by the traffic and the environmental conditions in which the task is performed. Although, obviously, traffic and environmental parameters are considered to have an effect on the cognitive system of the controllers (for example, these parameters affect their mental load, stress, etc.), in this approach, the behaviour of the cognitive system of the controller does not form part of the calculation of complexity.

\subsection{The Behavioural (Activity) Approach}

One could call the behavioural approach an attempt to include the ATCo in the calculation of complexity (see Fig. 1b). In this approach, complexity is defined and measured from the observable behaviour of the controller without any reference to the cognitive processing of traffic and environmental parameters. While it is assumed that controller behaviour is the result of cognitive processing of traffic and environment parameters, no attempt is made to model this cognitive processing. For example, the 
authors of [17] propose a method of measuring complexity from the actions of the controllers, and the authors of [18] have proposed another method where complexity is calculated from the commands issued by the controller.

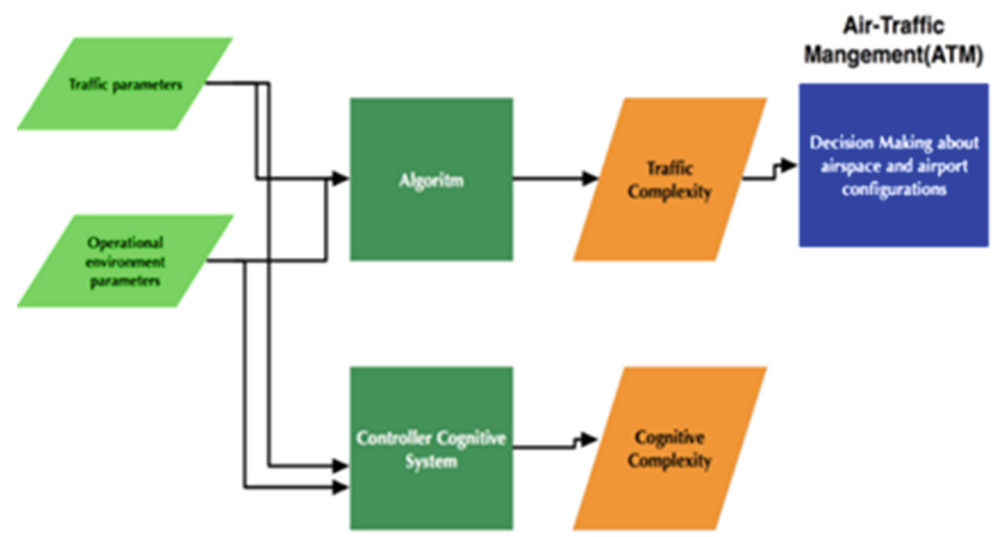

(a) Algorithm approach

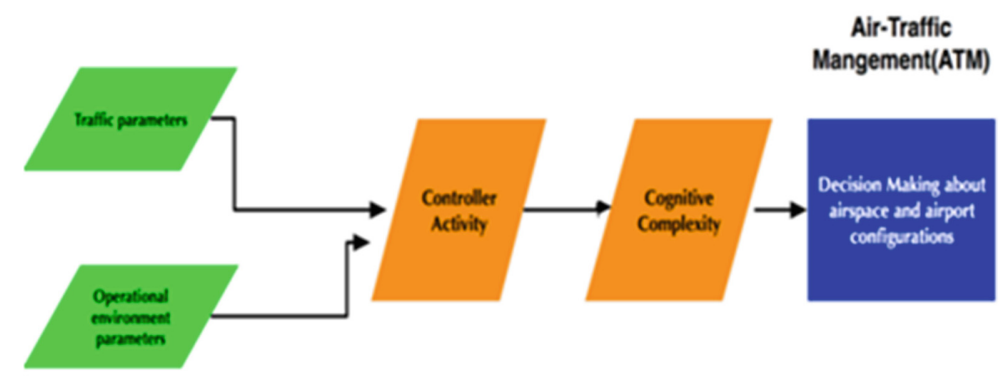

(b) Behavioural approach

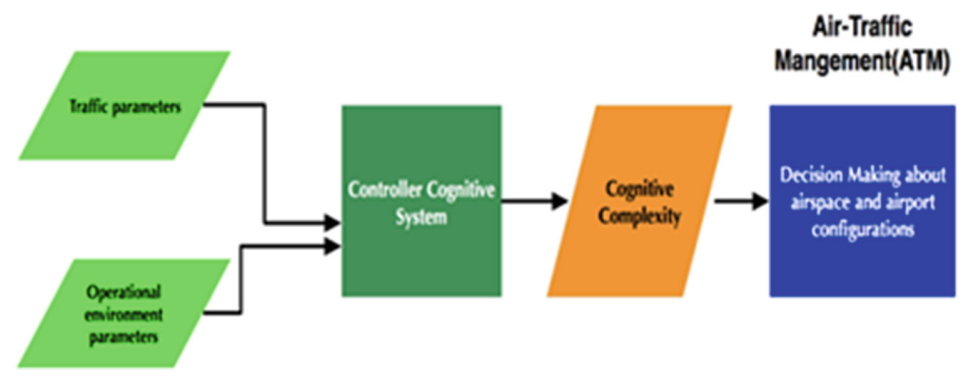

(c) Cognitive System approach

Fig. 1. The three approaches to the study of complexity in ATM 


\subsection{The Cognitive System Approach}

Finally, in the approach known as the cognitive system approach [19], complexity is calculated from the cognitive processing of traffic and environment parameters by the controller (see Fig. 1c). In this approach, the traffic and operational parameters are not important by themselves, but their importance is in the way the controller adjusts their decision-making strategies according to the parameters of traffic and environment.

This approach is built around the concept of a cognitive system. A cognitive system is one that performs the cognitive work of knowing, understanding, planning, deciding, problem-solving, analysing, synthesising, assessing, and judging as they are fully integrated with perceiving and acting. The characterisation of the airspace as a cognitive system represents a claim that the ATCo is part of an entity that does cognitive work, taking the parameters of traffic and operational environment into account. Other elements of the cognitive system are the artefacts and the environmental conditions within which the cognitive work of air-traffic control is carried out.

\section{Will Automation Always Reduce Complexity?}

The reduction of complexity has traditionally been addressed by introducing automatic systems into the task. From the algorithmic approach, the introduction of an automatic system is simply the assignment of a function that the person carried out before the introduction of the automatic system; however, in this reallocation of functions, it is not necessary to worry about cognitive processing nor the behaviour of the ATCo beyond the fact that a function has been taken from them and they have to do less things in the task [20]. In this way, it is assumed that the efficiency and safety of the system will always improve and no negative consequences of the introduction of automatic systems are predicted. In a similar way, from the behaviourist approach, the introduction of the automatic system can modify the behavioural strategies of the ATCo, but it is considered that this modification will reduce the complexity of the task, making the performance of the ATCo always more efficient and without expecting any negative consequences from its introduction. On the contrary, from the cognitive system approach, it is considered that introducing an automatic element into the cognitive system will affect the system as a whole, improving its efficiency, but also producing potentially negative effects. Some of these negative effects will be derived from the modification of the ATCo mental processing of the traffic and operational parameters and, as a consequence of that, from their behaviour.

The experience of controllers and the research done on this issue over many years show that, in fact, these negative effects exist as predicted by the cognitive system approach $[13,14]$. These negative effects could mainly be the consequence of a failure of the automatic system. The positive effects of automation in reducing the complexity of the task are obtained when the automatic system works well. However, the reliability of the automatic system is never one hundred percent and there will always be a small probability that the system will fail, affecting ATCo cognitive processing and 
behaviour. Neither the algorithmic nor the behaviouristic approaches would predict those negative consequences of automation failures.

For example, there is extensive literature on the well-known problem of being 'out of the loop' (OOTL). This problem occurs when the person, in our case the ATCo, is put out of the loop of the 'perceiving-acting-perceiving circle'. In a normal situation, a person is within a cycle of observation-action-feedback-action [21, 22]: the person observes the situation, acts on it, observes the results of their performance, and, if this is not correct or is insufficient, they act again to correct it. When the automatic system is introduced, the person is removed from that cycle by taking from them the functions of observing and receiving feedback on what the system is doing. If the system works well, removing the person from the loop will have no consequences; however, if the system fails, the person must note this failure and take control of the situation. The phenomenon of OOTL occurs when, after a change in the situation - in particular, high impact changes such as system failures - the person does not return to the loop either because they have not realised the failure or because they do not react in time to take control of the situation. This phenomenon is not taken into consideration by the algorithmic or behavioural approaches, but can be explained easily from the cognitive system approach.

It can also happen that a person experiences one or several failures of the automatic system and they enter a state of overexcitement that leads to panic and erratic behaviour. The authors of [23] have shown the importance of non-cognitive skills (for example, tolerance to stress) in the training of ATCos. If the automation fault experience affects the stress of the controllers, that stress will have a negative effect on their performance. As it has been demonstrated numerous times, over-activation affects cognitive processes, such as visual perception, that are essential in the task of air control [24].

Therefore, in line with the view of function allocation in the cognitive engineering perspective [19, 22], from the cognitive system approach it can be considered that automation will reduce the complexity of the task only if the negative effects are reduced. If, as hypothesised, automation produces negative effects in addition to positive ones, those negative effects will increase complexity by counteracting the reduction in complexity produced by the positive effects.

It is worth mentioning that the cognitive system approach can predict negative effects of both high complexity and low complexity. Neither of the other two approaches can predict these effects, since in both approaches it is assumed that automation always reduces the complexity and, therefore, the mental workload of the ATCo. However, much of the negative effects of automation are due to the reduction in mental workload after reducing the complexity of the system. As we will explain below, for the phenomenon known as OOTL, which occurs in situations of low mental workload, when we introduce automation to reduce complexity it is only possible to explain and predict it from the cognitive system approach. 


\section{AUTOPACE Proposal to Reduce Complexity by Considering the Negative Effects of Automation}

At AUTOPACE, the effects of automation are explained within the cognitive system approach by proposing hypotheses from a psychological model of the ATCo. In this psychological model there are two components: the functional structure of the ATCo's cognitive system and dynamic management of attentional resources.

\subsection{The Psychological Model of the ATCo}

At AUTOPACE, we propose a psychological model that has a structure which is the hypothesised cognitive system of the ATCo. In addition, the model includes a description of how the cognitive system functions by requiring attentional resources. It is very important to differentiate between these two aspects - the structure and the functioning of the system - in order to understand our hypotheses about the effects of automation on controller performance. Our hypotheses are developed in the context of the predicted future scenarios of automation.

The Functional Structure of the Cognitive System. All the cognitive models that have been proposed in the literature to explain the interaction between a person (i.e., the ATCo) and a system share the same scheme in which the human cognitive system is composed of sensory, perceptual, memory, and decision-making processes. We can take as a reference the model proposed in [25] (see Fig. 2). This model incorporates several interesting aspects of the recent theoretical developments in the science of human factors. In particular, we might highlight the incorporation in the model of the levels of processing that constitute what is called situation awareness (SA) [26]: perception, comprehension, projection, and decision.

Cognitive Functioning and Mental Resources. Human behaviour and mental activity require energy. In a sense, we could say that, in the life sciences, research has followed a mechanistic paradigm, according to which human machinery function depends on supplied energy [27]. Thus, for instance, it is assumed that performance of a task will improve or deteriorate depending, among other things, on the quantity and quality of the energy (resources) supplied [28]. In the tradition of attentional theories and human factors and ergonomics, this energy is called 'resources'.

Using a simple model of human functioning, we can say that when a person is confronted with the performance of a task, they do so with a certain amount of mental resources that we call available resources. The amount of resources that a person has depends on several factors, both individual and contextual. In addition to the available resources, we must talk about what we call demanded or required resources. Depending, essentially, on the complexity of the task, a person will need to apply more or less available resources to be able to perform it with a certain level of optimisation. Easy tasks will require fewer resources, while difficult tasks more resources. 


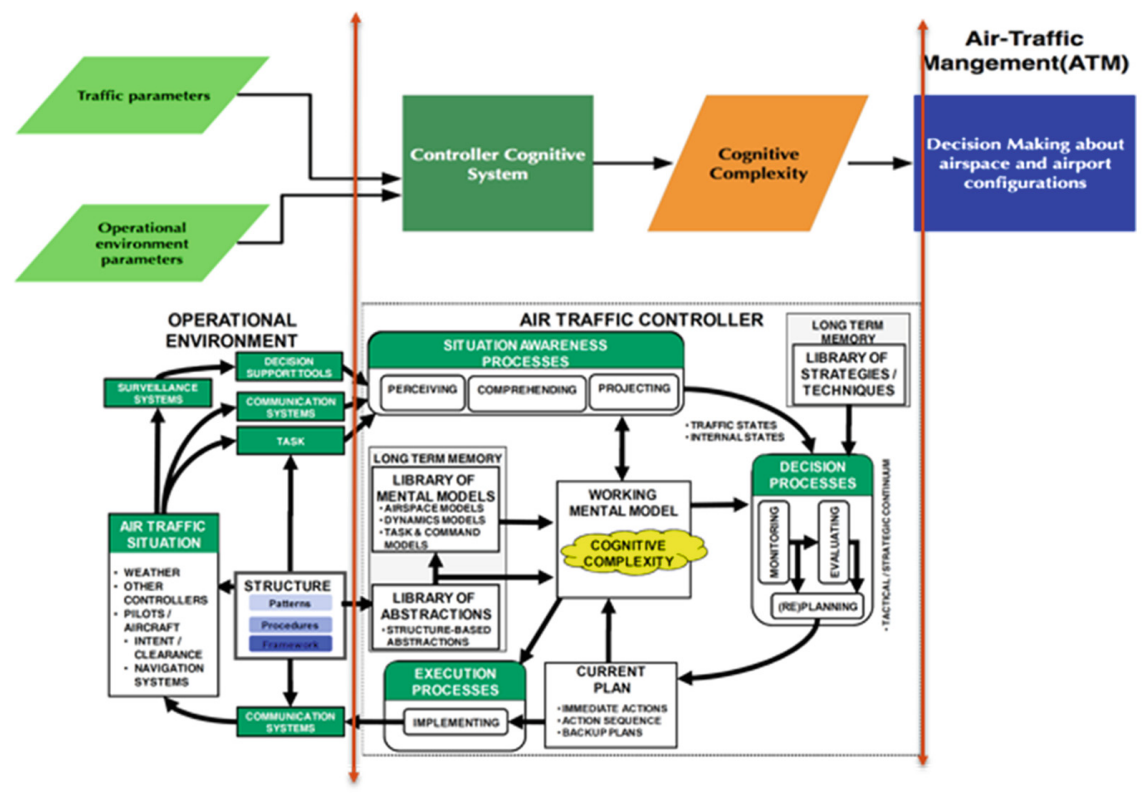

Fig. 2. The cognitive system approach and [25] model

Using these two concepts - demanded and available resources - psychological models have explained the functioning of the human cognitive system. The fundamental premise of all the models is that the functional structures, such as those described above, will work with an efficacy that will depend on the relationship between the demanded resources and the available resources. This relation is called the mental workload (MWL) [29-31] and is expressed according to formula 1:

$$
\text { MWL }=\frac{\text { Demanded_resources }}{\text { Available_resources }}
$$

The quantification of the cognitive demand resources can be made according to Wickens [32] and refined with reference to Wickens and McCarley [33]. When tasks overlap in time, the demanded resources depend on two factors: the resources demanded for processing for each cognitive channel (perception, comprehension, projection, decision-making, and manual or verbal actions) and the amount of interference between the two tasks. When two tasks are performed in parallel and use the same pool of resources, there would be interferences that increase the demanded resources. This increase could be reduced by the prioritisation of tasks. These two factors could be expressed as shown in Formula 2 [32]. In our model, this is the general formula used for calculating an ATCo's demanded resources. 


$$
\text { Demanded resources }=\sum_{c=1}^{u} w_{c}+\sum_{c=1}^{n} \sum_{d=c+1}^{N} i^{(c, d)}
$$

$\mathrm{w}_{\mathrm{c}}=$ resources demanded by channel: perception (visual, auditory), comprehension, projection, decision-making, response (manual or verbal)

$\mathrm{i}^{(\mathrm{c}, \mathrm{d})}=$ interference between channels $\mathrm{c}$ and $\mathrm{d}$.

This equation reflects the assumption that the demand for resources depends on the sum of the weights associated with the demand of the different cognitive channels involved in a task and the sum of the values of interference between channels. The second component of this formula represents the cost that the interference between channels has in the calculation of the resources demanded. Therefore, the traditional way of understanding complexity coincides with the definition of demanded resources. In this way, it is considered that the more complexity, the more mental resources will be required. In other words, complexity has traditionally been defined as the amount of mental resources that a task demands. However, the basic assumption of AUTOPACE is that automation also affects the available resources. We assume that a control situation may demand few mental resources, but it can become very complex because the ATCo does not have available resources necessary to face the demand of resources.

The available mental resources (the denominator of Formula 1) are considered to be a pool of resources that a person has at their disposal to perform a task. This pool could be made up of different dimensions containing more or less available resources. In the traditional view of human factors research, it has always been considered that the person performing a given task uses the whole pool of available resources. In the case where the available pool is small, the denominator in the equation for calculating mental workload is small, contributing to a major probability of overload. On the contrary, when the pool is large, the person would use all the resources at his disposal, making the denominator large and the probability of underload higher. The dimension of the pool of available resources depends on a number of factors, such as stress, fatigue, emotions, etc., all of which are factors that affect the level of activation or arousal.

However, this traditional view of the pool of available resources has been reviewed in a sense that is very relevant for AUTOPACE. It is increasingly recognised that not all the available resources that a person possesses need to be allocated to perform the task. For many different reasons, the proportion of the available resources allocated to the performance of a task may vary considerably, both during task performance and from one iteration to another of that same task. This new understanding of the available resources is behind the recent interests of researchers of concepts such as engagement or effort. For example, a recent paper [34] has reviewed the literature on effort. For more than one hundred years, psychologists have been working on the concept of effort to understand why and how a person dedicates more or less available resources to a task. In educational psychology, there is a well-known theoretical model called Cognitive Load Theory [35] in which there are three components that are differentiated:

1. Intrinsic load which is directly related to the learning material and that is what we call demanded resources in our context of human factors; 
2. Extraneous load which is the resources dedicated to other tasks, but not to the task of learning itself; and

3. Germane load, which refers to the mental resources devoted to acquiring and storing schemata in long-term memory (learning itself).

This third component refers to the mental resources that are in the pool of available resources at one point in time and actually allocated to the main task. This component of cognitive load is the issue of interest in [34]. The author realised that the available resources that are in the pool can be allocated to different tasks simultaneously and when we are calculating the total MWL of one task, we should enter in the denominator the real available resources that are dedicated to the task of our interest. For the same reason, researchers are talking more and more about engagement, another concept related to available resources and effort (i.e., [36]). It seems obvious that when performing a task, a person could be more or less engaged in it. Engagement affects the amount of resources that the person will make available to performing a task: the more engaged in the task, the more available resources are allocated to it. We can describe engagement as a continuum. At one end of the engagement scale, there is a 'passive cognitive engagement' that leads to allocating a small amount of resources. On the other end, there is an 'active cognitive engagement' that increases the amount of available resources allocated to the task. Therefore, engagement might affect the size of the pool of available resources, but more importantly, it would determine how much of those available resources are dedicated to the task. In that sense, engagement means something similar to germane load in educational psychology theories.

Our interest in these new interpretations of available resources for this project is clear: although we assume that automation reduces the demanded resources of the task, in our proposal, automation affects also the available resources in two ways:

1. The level of activation or arousal (psychophysiological activation), and, subsequently, the size of the pool of available resources; and

2. The amount of those available resources that are really dedicated to the task.

As we will explain in the following sections, the available resources that are allocated to the task will vary because ATCo responsibilities will change in the future automation scenarios. That means that there might be also a change in 'engagement' or 'effort' and, consequently, in the amount of available resources dedicated to the task.

In our proposal, these changes in responsibilities mean changes in available resources allocated to the task of control. Therefore, we will consider that automation will affect the available resources. An ATCo who is simply monitoring would be less engaged than another one who is approving or applying and, therefore the denominator of the MWL equation will be smaller (i.e., more risk of OOTL). Then, when the automatic system fails and the ATCo has to recover control, the probability of being out of the loop is higher when they are less engaged and dedicating less effort to the task. That explains why OOTL is more probable when monitoring than when approving or applying. Therefore, our hypotheses can be considered in relation to the attentional theories, as shown in Fig. 3. In the classical theories of attentional resources (i.e., [28]), automation affects only the demanded resources. These traditional theories are in line with the algorithmic and behavioural approaches to complexity; if 
automation affects only the demanded resources by reducing them, it is not necessary to take into account the human cognitive system because it directly benefits from changes made in the conditions of the traffic and the operational environment. The cognitive system benefits from automation, but it is not necessary to act on it. Although, it can be argued that knowing the functioning of the human cognitive system could be useful to better identify the aspects of traffic and the operational environment which would reduce the cognitive demands, the main assumption would be that any automatic system would have some positive benefit for the ATCo and no negative effects. However, when we consider the new attentional theories (i.e., [37]) which assume that automation also affects available resources, we must necessarily adopt the approximation of cognitive systems, since only from this approach is it considered that automation can modify the operation of the system as a whole (especially the interaction between the human cognitive system and the automatic system). For this reason, complexity cannot be calculated without considering the human cognitive system in its interaction with the automatic system.

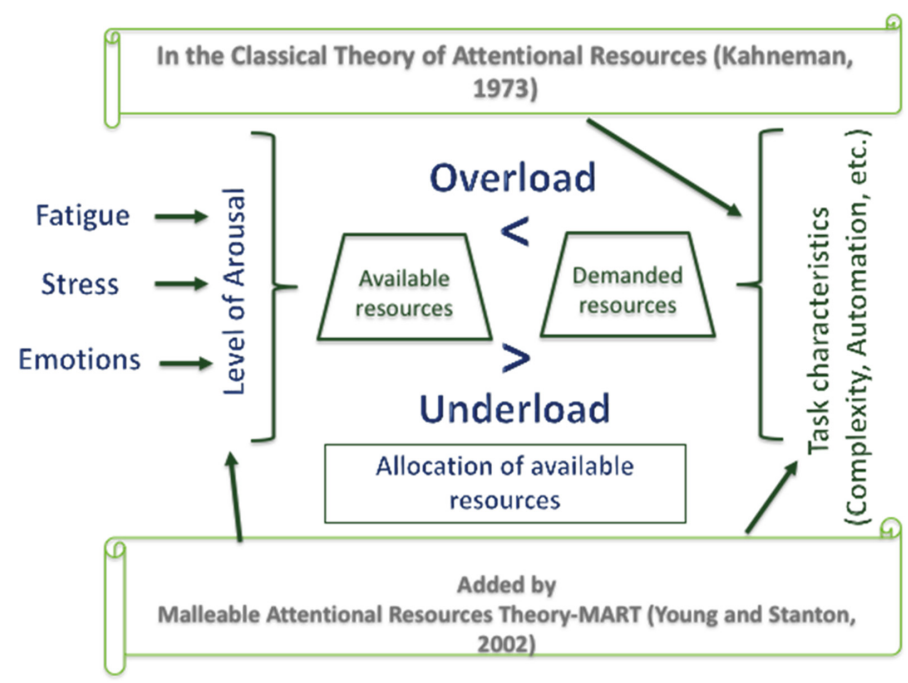

Fig. 3. Effects of automation according to the traditional and new attentional theories.

\subsection{AUTOPACE Psychological Model in the Context of Related Research on Mental Workload}

The psychological model proposed in AUTOPACE incorporates current theories and empirical results on mental workload [29-31]. The field of mental workload research has currently two major challenges. In the first place, the theoretical models proposed up to date should allow to make predictions in applied context where the mental workload of the operators is a fundamental factor to explain their performance. In this aspect, the research carried out in the AUTOPACE project demonstrates how the mental workload of the ATCo is fundamental to explain how she/he deals with the 
complexity of the control task. Secondly, although much progress has been made in these years, we are still in need of methodological development to validate the hypothesis derived from the theoretical models. In this second aspect, AUTOPACE contributes with a methodology based on the development of a computational model where the psychological model of the ATCo is implemented. We explain this methodology in the following section. But, first, we must describe in more detail the hypotheses that derive from the psychological model developed in AUTOPACE.

\section{AUTOPACE Predictions for Future Automation Scenarios in ATC}

In order to test our hypotheses, at AUTOPACE, we have defined the scenarios in which ATCos will work when automation is introduced in their work. We foresee two possible scenarios: one with medium automation and another with high automation. Those two scenarios will require different responsibilities and different levels of engagement with the task. We hope that by comparing these two scenarios, we can observe the behaviour of the ATC system when the psychological processes and the behaviour of the ATCos are affected by the different levels of automation. These two scenarios of future automation considered by AUTOPACE are described below.

The scenarios considered at AUTOPACE represent future traffic and mode of operations according to the SESAR Concept of Operations. The characteristics of this Concept of Operations are:

- It considers annual growth of $2.7 \%$ from 2015 to 2050 (an increase of $94.5 \%$ );

- It implements free route and 4D trajectory concepts;

- Trajectories are de-conflicted thanks to the implementation of de-complexing processes;

- Sectors are expected to be much bigger than current sectors; and

- Several ATCos will be operating in the same sector (flight centred ATS procedures).

AUTOPACE describes two different levels of automation that could be expected by 2050: high automation scenario (E1) and medium automation scenario (E2). These scenarios are defined by means of four scenario elements:

- Actors: the scenarios consider two actors as relevant for AUTOPACE purposes: the ATC System and the ATCo. Current executive and planner ATCo actors will not be needed thanks to automation. Therefore, AUTOPACE scenarios consider a unique human actor: the ATCo who will assume both executive and planner roles.

- Responsibilities allocated to actors: the responsibilities of each actor in each scenario is the most relevant factor for the cognitive modelling study. For this reason, we have defined three verbs to describe ATCo responsibilities with the following criteria:

- Monitor: When the ATC System assumes the major ATC actions, the ATCo must monitor system behaviour to prevent deviations. Monitoring or vigilance is the activity that an operator performs to acquire situation awareness (SA). Due to the high level of automation, the ATCo must monitor in both high and 
medium automation scenarios. It is important to note that the ATCo could not apply or approve actions without previously monitoring.

- Approve: Once the ATC System has proposed an ATC action, the ATCo must approve it before it is implemented. Approval requires previous monitoring, but also an evaluation of the correctness of the system decision. Approval does not imply the implementation of the action, but the ATCo must consider the consequences of the action carried out by the system. Therefore, we might say that approval requires a good SA (perception, comprehension, and projection, with projection being more relevant for approving than for monitoring).

- Apply. The ATCo analyses the situation, decides, and implements the most suitable solution from a set of provided ATC system solutions and with the support of the ATC tools. Application requires monitoring too, but, in contrast with approval, it is the ATCo who must elaborate the solution to the problem and then identify and implement the necessary actions to carry it out. Therefore, application should require not only SA (perception, comprehension, and projection), but also the use of decision-making and responding resources (verbal, manual).

- Processes and services: AUTOPACE ConOps identifies eight processes and describes the role that the ATCo plays in them for high automation and medium automation scenarios.

- Human performance aspects: a preliminary identification of the challenges that each scenario will have from the human factors perspective.

According to these criteria:

- In the high automation scenario, the ATCo is expected to have the responsibility of monitoring or monitoring and approving in the provision of the majority of the ATC services.

- In the medium automation scenario, the ATCo will be responsible not only for monitoring and approving, but also for applying many of the ATC services (after analysing the proposals made by the system).

Table 1 summarises the description of some of the ATC Controller responsibilities.

Table 1. Some responsibilities allocated to the ATC controller in the future automation scenarios

\begin{tabular}{l|l|l}
\hline Responsibilities & \multicolumn{2}{l}{$\begin{array}{l}\text { Responsibilities } \\
\text { allocation }\end{array}$} \\
\cline { 2 - 3 } & $\begin{array}{l}\text { High } \\
\text { automation }\end{array}$ & $\begin{array}{l}\text { Medium } \\
\text { automation }\end{array}$ \\
\hline Identify conflict risks between aircraft & Monitor & Monitor \\
\hline Provide flight information to all known flights & Monitor & Monitor \\
\hline Relay to pilots SIGMETS that may affect the route of a flight & Monitor & Monitor \\
\hline $\begin{array}{l}\text { Provide Alerting Service (ALRS) to all known flights according to } \\
\text { the following three different phases (INCERFA, ALERFA, }\end{array}$ & Monitor & Monitor \\
DETRESFA) & & \\
\hline
\end{tabular}


Table 1. (continued)

\begin{tabular}{|c|c|c|}
\hline \multirow[t]{2}{*}{ Responsibilities } & \multicolumn{2}{|c|}{$\begin{array}{l}\text { Responsibilities } \\
\text { allocation }\end{array}$} \\
\hline & $\begin{array}{l}\text { High } \\
\text { automation }\end{array}$ & $\begin{array}{l}\text { Medium } \\
\text { automation }\end{array}$ \\
\hline $\begin{array}{l}\text { Check flightplans/RBT/RMTs for possible conflicts and } \\
\text { complexity issues within area of responsibility }\end{array}$ & Monitor & Monitor \\
\hline Plan conflict-free flight path through area of responsibility & Monitor & Monitor \\
\hline $\begin{array}{l}\text { Provide early conflict detection and resolution if the early } \\
\text { resolution brings operational benefit (either on the ground side or } \\
\text { the airborne side) }\end{array}$ & Monitor & Approve \\
\hline Assign specified headings, speeds and levels & Monitor & Approve \\
\hline Re-route flights to avoid non-nominal or hazardous weather areas & Monitor & Approve \\
\hline Provide sequencing between controlled flights & Monitor & Approve \\
\hline Resolve boundary problems by re-coordination & Monitor & Approve \\
\hline $\begin{array}{l}\text { Implement solution strategies by communicating trajectory } \\
\text { changes to the aircraft through the concerned ATC } \\
\text { Controller/System via Data Link }\end{array}$ & Monitor & Approve \\
\hline Provide separation between controlled flights & Monitor & Apply \\
\hline $\begin{array}{l}\text { Apply appropriate separation to all controlled flights departing area } \\
\text { of jurisdiction }\end{array}$ & Monitor & Apply \\
\hline Monitor the air situation picture & Monitor & Apply \\
\hline Monitor the weather conditions & Monitor & Apply \\
\hline $\begin{array}{l}\text { Monitor information on airspace status e.g. activation of segregated } \\
\text { airspace Communicate with pilots by data link }\end{array}$ & Monitor & Apply \\
\hline Monitor aircraft equipment status as provided by the system & Monitor & Apply \\
\hline $\begin{array}{l}\text { Co-ordinate with adjacent control areas/sectors for the delegation } \\
\text { of airspace or aircraft }\end{array}$ & Monitor & Apply \\
\hline $\begin{array}{l}\text { In coordination with the ATC Supervisory or Local Traffic } \\
\text { Management roles determine the need for Complexity Solution } \\
\text { Measures in the case of overload situations forecast }\end{array}$ & Approve & Apply \\
\hline Issue holding instructions & Approve & Apply \\
\hline
\end{tabular}

\section{Methodology}

When complexity is approached from a theoretical psychological model of the ATCo, it is possible to derive hypotheses from that model about how to reduce complexity with automation. These hypotheses refer to how the complexity varies depending on how the ATCo processes the traffic parameters and how the operational environment is designed and managed. These hypotheses can also be made about the measures that can be put in place to mitigate these effects. Then, these hypotheses must be tested using a scientifically valid method. 


\subsection{The ATCo Psychological Model Implemented in a Computational Model}

There are several scientifically valid methods for testing hypotheses in a dynamic task. An alternative to the methods designed to obtain empirical data to test the hypotheses that are derived from a theoretical model, is the method that has been called the 'computational method' [38, 39], which consists of developing a computer model in which the psychological model is implemented. With this computer model, it is possible to run computer simulations where the hypothesis derived from the model can be tested. In order to do that, it is necessary that the computer model integrates the cognitive mechanisms responsible for the behaviour of the human actor, the task, and the environmental situation in which that task is performed. The hypotheses derived from the model are validated when the computer model responds in the way a human ATCo would respond when performing that task and in those traffic and environmental situations. For a recent review of this method and its applications for scientific discovery see [38].

At AUTOPACE, we have adopted this computational method. Subsequently, for the purpose of evaluating the effects of automation on the ATCo's cognitive system, we have employed a computational model prototype called COMETA (COgnitiveModEl for aTco workload Assessment), developed by CRIDA [40], that currently estimates the demanded resources required to perform the controller activity. The demanded resources are calculated based on the Wickens and McCarley algorithm [33]. Typically, COMETA inputs are the control events generated in real or simulation environments along with the ATCo task model expected in the scenario under study. For AUTOPACE, the control events have been generated by a fast-time simulation tool called RAMS. RAMS stands for 'reorganised ATC mathematical simulator'. This a FTS developed by ISA Software (http://ramsplus.com - taken on 06-03-2017)) where AUTOPACE Scenarios Environment (airspace and procedures) have been modelled. The ATCo task model (tasks associated with events, actions, behavioural primitives, and mental resources) has been adapted to the control activity expected in AUTOPACE

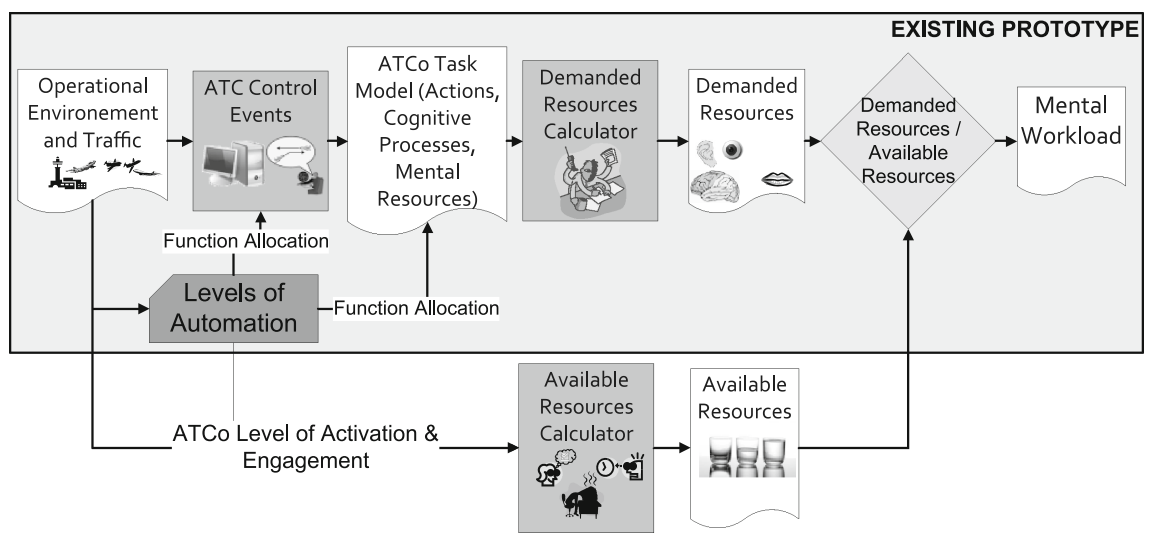

Fig. 4. COMETA functional architecture 
scenarios (high and medium automation) in nominal (when the system works well) and non-nominal situations (after system failures). COMETA presents the results not only as a global figure for the demanded resources, but also as an apportionment for every dedicated cognitive process and dimension.

COMETA foundations share the functional structure and functioning of the ATCo cognitive system models of AUTOPACE. Figure 4 shows the complete functional structure.

\subsection{Some Results to Validate the Predicted Effect of Automation on Mental Workload}

Figure 5 shows some results obtained with COMETA related to the functional structure evolution and the expected cognitive process in future automation scenarios, all of which were compared with the current ATC paradigm. As observed, the distribution of the functional structure of the cognitive system changes drastically with automation. While current ATCos use the cognitive dimensions (visual, comprehension, projection, decision-making, and verbal resources) in a balanced way, future ATCos will focus their cognitive efforts mainly on comprehension and projection. The ATCo needs to project what is going to happen in order to understand the system performance without missing situational awareness. In a medium automation scenario where main actions are not only monitored and approved, but also applied, projection is more relevant than comprehension as the ATCo needs to invest more resources into the projection of future scenarios to correctly select among the options given by the system (approve) and their own instructions (apply). In the high automation scenario, the contrary occurs and what is important is to have a more robust mental picture of what is occurring, in order to monitor system performance (monitor) and to approve system proposals (approving); i.e., better comprehension than projection. These results are in line with the predictions of different levels of automation made by [41].
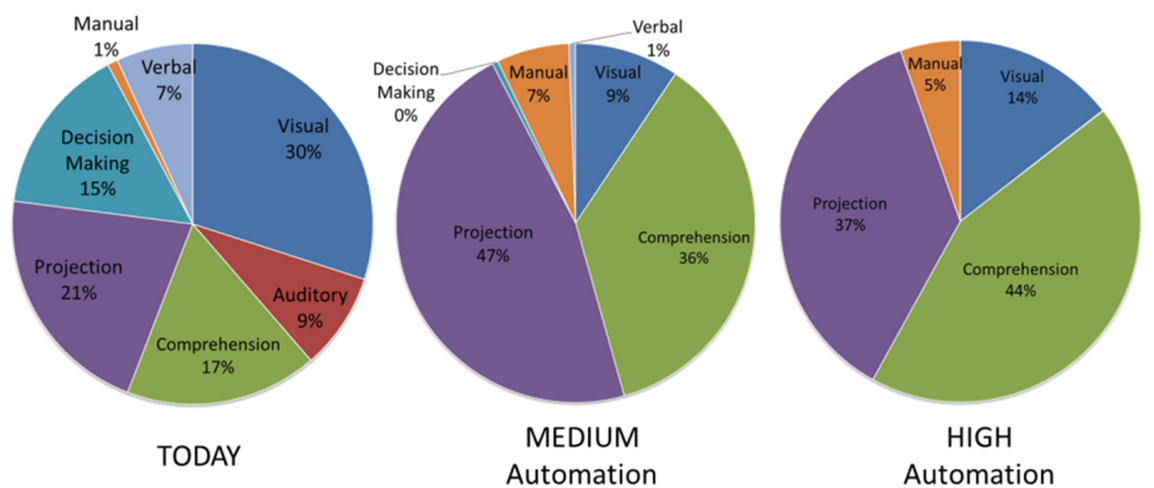

Fig. 5. Cognitive processes evolution in the current medium automation and high automation scenarios [42]. 


\section{Summary and Conclusions}

One of the main conclusions of Project AUTOPACE is that from a cognitive system approach to ATC complexity, it is possible to address the positive and negative effects of automation and propose measures to reduce its negative effects and improve system reliability and safety. Only from this approach is it possible to mitigate the negative effects of automation. The two other approaches do not predict negative effects since they consider that automation reduces the resources demanded (complexity is only dependent on the demanded resources). For this reason, in these approaches, many of the complex dimensions of human functioning are potentially ignored. An important consequence is that if the effects of automation are only considered with regard to traffic conditions and operational conditions, it is not possible to investigate the impacts of such profound transformations on human psychological processing and to adapt the way the human operator is prepared to manage such changes in their tasks. Therefore, only from the cognitive system approach is it possible to design training programs where cognitive and non-cognitive skills are taken into account in order to face the potentially negative effects of automation. It is very important to emphasise that when proposing a psychological model of the effects of automation, this model should be grounded in the consideration of mental workload because it is the psychological concept that defines the dynamic relation between the demanded and the available resources.

The ultimate goal of AUTOPACE is to indicate requirements for training competences resulting from the analysis carried out into the effect of automation on the ATCo psychological model. In future automation scenarios, some new training strategies and competences will be needed to cope with the effects of OOTL, stress, disorientation, panic, etc., to ensure that the ATCo performance is optimum. Therefore, the research carried out by AUTOPACE based on the ATCo psychological model will support future research on system design to balance the use of the different cognitive and non-cognitive processes and new training strategies to cope with the potentially negative effects of automation.

\section{References}

1. Osman, M.: Controlling uncertainty: a review of human behavior in complex dynamic environments. Psychol. Bull. 136, 65-86 (2010). https://doi.org/10.1037/a0017815

2. Broadbent, D.E.: Levels, hierarchies, and the locus of control. Q. J. Exp. Psychol. 29, 181201 (1977). https://doi.org/10.1080/14640747708400596

3. Dörner, D., Funke, J.: Complex problem solving: what it is and what it is not. Front. Psychol. 8, 1153 (2017). https://doi.org/10.3389/fpsyg.2017.01153

4. Frensch, P.A., Funke, J.: Complex Problem Solving: The European Perspective. Psychology Press, New York (2014). ISBN 0-8058-1336-5

5. Gopher, D., Donchin, E.: Workload: an examination of the concept. In: Boff, K.R., Kaufman, L., Thomas, J.P. (eds.) Hand-Book of Perception and Performance Cognitive Processes and Performance, vol. 2, pp. 41-49. Wiley, New York (1986). ISBN-13: 9780471829577 
6. Byrne, A.: Mental workload as an outcome in medical education. In: Longo, L., Leva, M.C. (eds.) H-WORKLOAD 2017. CCIS, vol. 726, pp. 187-197. Springer, Cham (2017). https:// doi.org/10.1007/978-3-319-61061-0_12

7. Longo, L.: Designing medical interactive systems via assessment of human mental workload. In: 2015 IEEE 28th International Symposium Computer-Based Medical Systems (CBMS), pp. 364-365. IEEE Press (2015). https://doi.org/10.1109/CBMS.2015.67

8. Balfe, N., Crowley, K., Smith, B., Longo, L.: Estimation of train driver workload: extracting taskload measures from on-train-data-recorders. In: Longo, L., Leva, M. (eds.) HWORKLOAD 2017. CCIS, vol. 726, pp. 106-119. Springer, Cham (2017). https://doi. org/10.1007/978-3-319-61061-0_7

9. Longo, L.: Mental workload in medicine: foundations, applications, open problems, challenges and future perspectives. In: 2016 IEEE 29th International Symposium ComputerBased Medical Systems (CBMS), pp. 106-111. IEEE Press (2016)

10. Tong, S., Helman, S., Balfe, N., Fowler, C., Delmonte, E., Hutchins, R.: Workload differences between on-road and off-road manoeuvres for motorcyclists. In: Longo, L., Leva, M. (eds.) International Symposium on Human Mental Workload: Models and Applications, pp. 239-250. Springer, Cham. (2017). https://doi.org/10.1007/978-3-319-61061-0_16

11. Edwards, T., Martin, L., Bienert, N., Mercer, J.: The relationship between workload and performance in air traffic control: exploring the influence of levels of automation and variation in task demand. In: Longo, L., Leva, M. (eds.) H-WORKLOAD 2017. CCIS, vol. 726, pp. 120-139. Springer, Cham (2017). https://doi.org/10.1007/978-3-319-61061-0_8

12. Loft, S., Sanderson, P., Neal, A., Mooij, M.: Modelling and predicting mental workload in en route air traffic control: critical review and broader implications. Hum. Factors 49, 376399 (2007). https://doi.org/10.1518/001872007X197017

13. Parasuraman, R., Riley, V.: Humans and automation: use, misuse, disuse, abuse. Hum. Factors 39, 230-253 (1997). https://doi.org/10.1518/001872097778543886

14. Metzger, U., Parasuraman, R.: automation in future air traffic management: effects of decision aid reliability on controller performance and mental workload. Hum. Factors 47, 35-49 (2005). https://doi.org/10.1518/0018720053653802

15. Mitchell, M.: Complexity: A guided tour. Oxford University Press, Oxford (2009). ISBN-13: 978-0199798100

16. Netjasov, F., Janić, M., Tošić, V.: Developing a generic metric of terminal airspace traffic complexity. Transportmetrica 7(5), 369-394 (2011). https://doi.org/10.1080/18128602. 2010.505590

17. Zhang, M., Shan, L., Zhang, M., Liu, K., Yu, H., Yu, J.: Terminal airspace sector capacity estimation method based on the ATC dynamical model. Kybernetes 45, 884-899 (2016). https://doi.org/10.1108/K-12-2014-0308

18. Tobaruela, G., Schuster, W., Majumdar, A., Ochieng, W.Y., Martinez, L., Hendrickx, P.: A method to estimate air traffic controller mental workload based on traffic clearances. J. Air Transp. Manag. 39, 59-71 (2014). https://doi.org/10.1016/j.jairtraman.2014.04.002

19. Kontogiannis, T., Malakis, S.: Cognitive Engineering and Safety Organization in Air Traffic Management. CRC Press, Boca Raton (2017). ISBN 9781138049727

20. Fitts, P.M.: Human Engineering for an Effective Air-navigation and Traffic-control System. National Research Council, Washington (1951)

21. Neisser, U.: Cognition and Reality: Principles and Implications of Cognitive Psychology. WH Freeman/Times Books/Henry Holt \& Co (1976). ISBN-13: 978-0716704775

22. Hollnagel, E., Bye, A.: Principles for modelling function allocation. Int. J. Hum.-Comput. Stud. 52, 253-265 (2000). https://doi.org/10.1006/ijhc.1999.0288 
23. Chappelle, W., Thompson, W., Goodman, T., Bryan, C.J., Reardon, L.: The utility of testing noncognitive aptitudes as additional predictors of graduation from US air force air traffic controller training. Aviat. Psychol. Appl. Hum. Factors 5, 93-103 (2015). https://doi.org/10. 1027/2192-0923/a000082

24. Woods, A.J.: The consequences of hyper-arousal for human visual perception. Retrieved from Dissertations \& Theses @ George Washington University (2010)

25. Histon, J.M., Hansman, R.J.: Mitigating complexity in air traffic control: the role of structure-based abstractions. Report no. ICAT-2008-05 (2008)

26. Endsley, M.R.: Toward a theory of situation awareness in dynamic systems. Hum. Factors: J. Hum. Factors Ergon. Soc. 37, 32-64 (1995). https://doi.org/10.1518/001872095779049543

27. Rabinbach, A.: The Human Motor: Energy, Fatigue, and the Origins of Modernity. University of California Press, Berkeley (1990). ISBN-13: 978-0520078277

28. Kahneman, D.: Attention and effort, Englewood Cliffs. Prentice-Hall, NJ (1973). ISBN-13: 978-0130505187

29. Longo, L., Leva, M.C. (eds.): Human Mental Workload: Models and Applications: First International Symposium, H-WORKLOAD 2017, Dublin, Ireland, June 28-30, 2017, Revised Selected Papers, vol. 726. Springer, Heidelberg (2017). https://doi.org/10.1007/9783-319-61061-0. ISBN 978-3-319-61061-0

30. Hancock, P.A.: Whither workload? Mapping a path for its future development. In: Longo, L., Leva, M. (eds.) H-WORKLOAD 2017. CCIS, vol. 726, pp. 3-17. Springer, Cham (2017). https://doi.org/10.1007/978-3-319-61061-0_1. ISBN 978-3-319-61061-0

31. Wickens, C.D.: mental workload: assessment, prediction and consequences. In: Longo, L., Leva, M. (eds.) H-WORKLOAD 2017. CCIS, vol. 726, pp. 18-29. Springer, Cham (2017). https://doi.org/10.1007/978-3-319-61061-0_2. ISBN 978-3-319-61061-0

32. Wickens, C.D.: Multiple resources and performance prediction. Theor. Issues Ergon. Sci. 3, 159-177 (2002). https://doi.org/10.1080/14639220210123806

33. Wickens, C.D., McCarley, J.S.: Applied Attention Theory. CRC Press, Boca Raton (2007). ISBN 9780805859836

34. Wickens, C.D.: Effort in human factors performance and decision making. Hum. Factors 56 (8), 1329-1336 (2014). https://doi.org/10.1177/0018720814558419

35. Sweller, J.: Cognitive load during problem solving: effects on learning. Cogn. Sci. 12, 257285 (1988). https://doi.org/10.1207/s15516709 $\operatorname{cog} 1202 \_4$

36. Endsley, M.: From here to autonomy: lessons learned from human-automation research. Hum. Factors 59(1), 5-27 (2017). https://doi.org/10.1177/0018720816681350

37. Young, M.S., Stanton, N.A.: Malleable attentional resources theory: a new explanation for the effects of mental underload on performance. Hum. Factors 44, 365 (2002). https://doi. org/10.1518/0018720024497709

38. Sozou, P.D., Lane, P.C., Addis, M., Gobet, F.: Computational scientific discovery. In: Magnani, L., Bertolotti, T. (eds.) Springer Handbook of Model-Based Science. SH, pp. 719734. Springer, Cham (2017). https://doi.org/10.1007/978-3-319-30526-4_33. ISBN 978-3319-30526-4

39. Rizzo, L., Dondio, P., Delany, S.J., Longo, L.: Modeling mental workload via rule-based expert system: a comparison with NASA-TLX and workload profile. In: Iliadis, L., Maglogiannis, I. (eds.) Artificial Intelligence Applications and Innovations. AIAI 2016. IFIP Advances in Information and Communication Technology, vol. 475, pp. 215-229. Springer, Cham (2016). https://doi.org/10.1007/978-3-319-44944-9_19

40. Suárez, N., López, P., Puntero, E., Rodriguez, S.: Quantifying air traffic controller mental workload. Fourth SESAR Innovation Days (2014) 
41. Endsley, M.R.: Level of automation effects on performance, situation awareness and workload in a dynamic control task. Ergonomics 42, 462-492 (1999). https://doi.org/10. $1080 / 001401399185595$

42. Cañas, J.J., Ferreira, P.N.P., Puntero, E., López, P., López, E., Gomez-Comendador, V.F.: An air traffic controller psychological model with automation. In: 7th EASN International Conference: "Innovation in European Aeronautics Research", Warsaw, Poland (2017) 